Published in final edited form as:

J Steroid Biochem Mol Biol. 2006 December ; 102(1-5): 97-102.

\title{
AROMATASE AND BREAST CANCER
}

\author{
A. Brodie, G. Sabnis, and D. Jelovac \\ Department of Pharmacology and Experimental Therapeutics University of Maryland School of \\ Medicine, Baltimore, MD 21201
}

\begin{abstract}
Several aromatase inhibitors and also new antiestrogens are now available for treating breast cancer. We have developed a model to compare the antitumor efficacy of these agents and to explore strategies for their optimal use. Results from the model have been predictive of clinical outcome. In this model, tumors are grown in ovariectomized, immunodeficient mice from MCF-7 human breast cancer cells transfected with the aromatase gene (MCF-7Ca). The possibility that blockade of estrogen action and estrogen synthesis may be synergistic was explored by treating mice with the aromatase inhibitor letrozole and the antiestrogen tamoxifen alone and in combination. The results indicated that letrozole alone was better than all other treatments. In addition, when tamoxifen treatment was no longer effective, tumor growth was significantly reduced in mice switched to letrozole treatment. However, tumors ultimately began to grow during continued treatment. To investigate the mechanisms by which tumors eventually adapt and grow during Ietrozole treatment, we determined the expression of signaling proteins in tumors during the course of letrozole treatment compared to the tumors of control mice. Tumors initially upregulated the ER while responding to treatment, but subsequently receptor levels decreased in tumors unresponsive to letrozole. Also, Her-2 and adapter proteins (p-Shc and Grb-2) as well as all of the signaling proteins in the MAPK cascade (p-Raf, p-Mekl/2, and p-MAPK), but not in the Pl3/Akt pathway, were increased in tumors no longer responsive to letrozole. To investigate whether sensitivity to letrozole could be regained, cells were isolated from the letrozole resistant tumors (LTLT) and treated with inhibitors of the MAPKinase pathway (P098059 and U0126). These compounds reduced MAPK activity and increased ER expression. EGFR/Her-2 inhibitors, gefitinib and AEE78S although not effective in the parental MCF-70a cells, restored the sensitivity of LTLT cells to letrozole. In xenografts, beginning treatment with letrozole and faslodex to down regulate the ER prevented increases in Her-2 and activation of MAPK and was highly effective in inhibiting tumor growth throughout 29 weeks of treatment. These results suggest that blocking both ER- and growth factor-mediated transcription may delay development of resistance and maintain growth inhibition of ER+ breast cancer.
\end{abstract}

\section{Introduction}

The majority of breast cancer patients are postmenopausal women with estrogen receptor positive $(\mathrm{ER}+)$ tumors that are responsive to the mitogenic effects of estrogen. In these patients, estrogen is no longer synthesized in the ovary but in other tissues such as adipose tissue and breast tissue. Despite low circulating estrogen levels, the concentration of estrogen in the breast of postmenopausal patients is several fold-higher and equal to that of premenopausal women. This suggests that estrogen produced in or near the tumor could have a significant impact on its progression.

\footnotetext{
Publisher's Disclaimer: This is a PDF file of an unedited manuscript that has been accepted for publication. As a service to our customers we are providing this early version of the manuscript. The manuscript will undergo copyediting, typesetting, and review of the resulting proof before it is published in its final citable form. Please note that during the production process errors may be discovered which could affect the content, and all legal disclaimers that apply to the journal pertain.
} 
Inhibiting the effects of peripherally formed estrogen can be achieved either by blocking the estrogen receptor with antiestrogens or blocking the synthesis of estrogens with aromatase inhibitors. To date, the antiestrogen, tamoxifen has had a major impact on the treatment of breast cancer. More recently, three aromatase inhibitors have become available in the US. The aromatase inhibitors approved for breast cancer treatment are nonsteroidal triazole compounds, letrozole and anastrozole, and exemestane which is a steroidal analogue of androstenedione. Current indications are that all three are more beneficial than tamoxifen, possibly because they are not associated with weak agonistic properties and therefore exert more complete reduction in estrogenic effects than tamoxifen. In addition to tamoxifen, the antiestrogen fulvestrant has recently been approved for treatment of advanced breast cancer. This compound downregulates the ER. However, many questions remain to be answered concerning the most strategic use of these compounds.

To compare the effectiveness of aromatase inhibitors and antiestrogens we have developed a tumor model $[1,2]$ using human hormone-responsive (ER+) breast cancer cells stably transfected with the human aromatase gene (MCF-7Ca) [3]. These MCF-7Ca cells serve as an autocrine source of estrogen that stimulates the cells to form tumors when inoculated into ovariectomized, immunesuppressed mice and grow as tumors. The resulting tumors are sensitive to both the antiproliferative effects of antiestrogens and aromatase inhibitors [1,2]. The model compares with the postmenopausal breast cancer patient as the source of estrogen after menopause is from non-ovarian tissue, and is not regulated by gonadotropins.

This model has proved valuable to study strategies of treatment with aromatase inhibitors and antiestrogens. We have investigated whether the two types of agents by inhibiting estrogen action as well as synthesis could be more effective in combination than either aromatase inhibitor or antiestrogen alone. We have also investigated whether the tumor growth inhibition can be prolonged by using these agents in sequence [4-7].

\section{Materials and Methods}

Letrozole (CGS 20267) was kindly provided by Dr. D. Evans (Novartis Pharma A.G., Basel, Switzerland). The pure antiestrogen fulvestrant (ICI 182,780) was generously supplied by Drs. A. Wakeling and E. Anderson (AstraZeneca Pharmaceuticals, Macclesfield, United Kingdom). Exemestane was provided by Pharmacia (Pfizer Pharmaceutical, Groton, CT). Tamoxifen and $0.3 \%$ hydroxypropyl cellulose was purchased from Sigma Co. (St. Louis, MO).

\section{MCF-7Ca Breast Cancer Cells}

MCF-7 human breast cancer cells stably transfected with the human aromatase gene (MCF-7Ca) were kindly provided by Dr. S. Chen, City of Hope, Duarte, CA [3] and routinely maintained in DMEM with 5\% fetal bovine serum, 1\% penicillin/streptomycin solution, and $750 \mu \mathrm{g} / \mathrm{mL}$ G418.

\section{MCF-7Ca Tumor Xenograft Models}

Female ovariectomized BALB/c athymic nude mice 4 to 6 weeks of age were obtained from the National Cancer Institute (Frederick, MD). The animals were housed in a pathogen-free environment under controlled conditions of light and humidity and received food and water ad libitum. All animal studies were carried out according to the guidelines approved by the Animal Care Committee of the University of Maryland School of Medicine.

For inoculation, subconfluent MCF-7Ca cells were suspended in Matrigel $(10 \mathrm{mg} / \mathrm{mL})$ at 2.5 $\times 107$ cells $/ \mathrm{mL}$. Mice were injected s.c. with $100 \mu \mathrm{L}$ of cell suspension in two sites per flank. All animals received a daily supplement s.c. of androstenedione $(100 \mu \mathrm{g} / \mathrm{d}$; aromatase 
substrate) throughout the experiment. Tumor volumes were measured weekly as reported previously [1,2]. Treatments began when the tumors reached a measurable size $\left(\sim 300 \mathrm{~mm}^{3}\right)$. Mice were assigned to groups for treatment, so that there was no statistically significant difference in tumor volume among the groups at the beginning of treatment. Mice were injected s.c. daily with the drugs in $0.3 \%$ hydroxypropyl cellulose. At the times indicated in the figures, mice were killed by decapitation and the trunk blood was collected. Tumors and uteri were excised, cleaned, weighed, and stored in liquid nitrogen for analysis later [6,7].

\section{Long-term letrozole treated tumor cells (LTLT-Ca)}

Tumor cells were isolated from MCF-7CA tumors of mice treated with letrozole for 56 weeks [7]. Tumors were cut into small pieces $\left(0.5 \mathrm{~cm}^{3}\right)$, then further disrupted by being repeatedly drawn into a pipette. The tumor cells were centrifuged for $5 \mathrm{~min}$ at $1000 \mathrm{rpm}$. The cells were incubated at $37^{\circ} \mathrm{C}$, stirring overnight with collagenase type $1-\mathrm{A}(1 \mathrm{mg} / \mathrm{ml})$ and hyaluronidase type $1-\mathrm{S}(1 \mathrm{mg} / \mathrm{ml})$ in RPMI-1640 medium with 5\% FBS, $2 \times$ penicillin/streptomycin, $2.5 \mu \mathrm{g} /$ $\mathrm{ml}$ amphotericin B, $50 \mathrm{U} / \mathrm{ml}$ polymixin B sulfate and $50 \mu \mathrm{g} / \mathrm{ml}$ gentamicin sulfate. The following day, cells were washed three times with DPBS and centrifuged and filtered sequentially through a $70 \mu \mathrm{m}$ filter followed by a $40 \mu \mathrm{m}$ filtration. After the last filtration, cells were suspended in phenol red-free IMEM media supplemented with 10\% CSS, $2 \%$ penicillin/ streptomycin solution, $5.0 \mu \mathrm{g} / \mathrm{ml}$ amphotericin B solution, and $50 \mathrm{U} / \mathrm{ml}$ polymixin B and plated into $2 \mathrm{~cm} \times 10 \mathrm{~cm}$ petri dishes. The following day, the dishes were washed three times with DPBS and the media refreshed. One week later, the plates were lightly washed with phenol red-free trypsin/versene solution and then washed with DPBS to remove any stromal cells. The cells were maintained in phenol red-free IMEM media supplemented with 10\% CSS, $2 \%$ penicillin/streptomycin solution, $2.5 \mu \mathrm{g} / \mathrm{ml}$ amphotericin B solution, $600 \mu \mathrm{g} / \mathrm{ml} \mathrm{G} 418$, and 1 $\mu \mathrm{M}$ letrozole. The medium was refreshed twice a week until colonies started to proliferate. Over the next 2-3 weeks, serum levels in the medium were reduced from 10 to 5\% CSS, penicillin/streptomycin level from 2 to $1 \%$, and amphotericin was removed. Rapidly growing colonies were selected and each colony added to 1 well of a 6-well plate in phenol red-free IMEM supplemented with 5\% CSS, $1 \%$ penicillin/streptomycin, $600 \mu \mathrm{g} / \mathrm{ml} \mathrm{G} 418$, and $1 \mu \mathrm{M}$ letrozole.

\section{Western Blotting}

Cell lysates and protein extracts from tumor tissues were prepared by homogenizing the cells and tissue in lysis buffer. Proteins in the lysates were separated on a denaturing polyacrylamide gel and transferred to a nitrocellulose membrane. The protein-bound membranes were then incubated for 1 hour at room temperature with $0.1 \%$ Tween 20 in PBS (PBS-T) and 5\% nonfat dried milk to block non-specific binding to antibodies. The membranes were then incubated with respective primary antibodies in PBS-T milk for 1 hour, and specific binding was visualized by using species-specific $\mathrm{IgG}$ followed by enhanced chemiluminescent detection (ECL kit; Amersham) and exposure to ECL X-ray film [8].

\section{Statistical analysis}

Linear mixed-effects models were fitted to estimate average tumor volume within each treatment group. Each experiment was analyzed separately. The mean effect of treatment in each group and random effects for each mouse within a group were estimated. Data on tumor volume were longitudinal and unbalanced. The duration of treatment varied across the treatment groups. It was not possible to measure the same number of multiple tumors per mouse at all time points. In addition, diagnostic plots suggested that models of exponential growth were appropriate to the tumor growth data. Therefore, linear mixed-effects models were fitted to the natural logarithm of tumor volume over time. This approach allows an exponential 
parameter controlling the rate of growth to be estimated for each of these treatment groups, with the random effects being estimated for each subject in a group [9].

All hypothesis tests were two-sided. Adjustments for multiple comparisons were made by using either Tukey's or Dunnett's procedure [10,11]. Results are presented as treatment mean \pm S.E. and compared at the 0.05 level of significance.

\section{Results and Discussion}

\section{Antitumor efficacy of aromatase inhibitor and antiestrogen treatment in MCF-7 aromatase xenografts}

Combined Treatment Letrozole and Tamoxifen-The tumor model was also utilized to determine whether combining an agent that inhibited estrogen synthesis with one that inhibited estrogen action may have synergistic actions and be more effective than treatment with each agent alone. However, treatment with the combination of letrozole and tamoxifen resulted in tumor suppression similar to treatment with tamoxifen alone, but was less effective than letrozole alone. A similar observation had been made with anastrozole in combination with tamoxifen indicating that treatment was not improved by either of the two non-steroidal inhibitors combined with tamoxifen [5]. This result is reflected in the ATAC Trial where breast cancer patients did not benefit significantly more from the combined treatment of anastrozole and tamoxifen than tamoxifen alone [12].

Sequential Treatment: Letrozole and Tamoxifen or Fulvestrant-When mice were treated first with tamoxifen and then switched to second line treatment with letrozole, the reduction in tumor growth proved that treatment with the aromatase inhibitor was significantly more effective than continuing treatment with tamoxifen [6]. Recent clinical studies have confirmed the finding that sequential aromatase inhibitor treatment is more effective than continued tamoxifen [13]. Nevertheless, in the tumor model this strategy proved inferior to treatment with letrozole as the initial treatment. Tumors of mice treated with letrozole $(10 \mathrm{~g} /$ day) initially regressed and then began to grow slowly and took more than twice as long as tumors of mice treated with tamoxifen to double in volume [6].

In another study, mice with tumors that had started growing during letrozole treatment were then assigned to three groups so that each group had similar mean tumor volumes at the start of second-line treatment [6]. Group 1 received tamoxifen, Group 2 received fulvestrant, and Group 3 remained on letrozole. Both tamoxifen and fulvestrant were ineffective as second-line therapy following letrozole treatment [6]. The results suggest that switching from letrozole (10 $\mu \mathrm{g} / \mathrm{day}$ ) to antiestrogens is not an optimal choice for patients with tumors progressing on a therapeutically effective dose of letrozole.

Long-Term Letrozole Treatment-Despite the impressive antitumor efficacy of letrozole alone in the xenograft model, eventually tumors began to grow despite continued letrozole treatment. The ability of tumor cells to adapt and survive treatment is a major challenge to all cancer therapy. Thus, in order to investigate the adaptive changes that results in resistance to treatment, tumors were collected from mice at different times during the course of letrozole treatment. At 4 weeks, the tumors collected were regressing in response to treatment whereas at 28 weeks, tumors had grown back to their starting volume and had almost reached a doubling of tumor volume. Tumors collected at 56 weeks were continuing to grow and had more than doubled their volume even though letrozole treatment was maintained.

Increase in the estrogen receptor concentration is a likely mechanism that could allow the tumor to proliferate during letrozole treatment and could increase transcription despite low concentrations of ligand. Measurement of ER expression in tumor samples revealed that indeed 
ER was increased at 4 weeks of letrozole treatment. However, ER levels had decreased to below the control level at 28 weeks and further declined to low levels by 56 weeks. Nevertheless, p-ER (Ser167) was increased suggesting that ligand independent activation of ER may enhance proliferation in tumors treated with letrozole. The possibility that growth factors may have a role in increasing cell proliferation was investigated by Western blots. Tyrosine kinase receptor HER-2 expression was increased two-fold in the tumors treated with letrozole, not only at 28 and 56 weeks but also at 4 weeks when tumors were regressing. The expression of other proteins downstream of HER-2 was also increased. Grb-2 and p-MAPK were increased markedly in tumors treated for 28 and 56 weeks (6-fold) although slight increases were also seen at 4 weeks of treatment [8].

To carry out further studies, cells were isolated from the Long-Term Letrozole Treated (LTLT) tumors collected at 56 weeks. These LTLT cells formed tumors in nude mice that grew independently of estrogen or androstenedione and were not inhibited by letrozole. Compared to MCF-7Ca cells that adapted to grow in estrogen deprived medium (UMB-1 cells), ER expression in LTLT cells was decreased below the control level whereas HER-2, Grb-2, pSchc, p-Raf, pMER1/2, p-p90 RSK and p-elk were all up-regulated 2-6-fold. In contrast, proteins in the PI3K/Akt pathway were not increased in the LTLT cells but were increased in the UMB-1 cells along with ER and HER-2.

Further evidence that the MAPK pathways enhanced LTLT cell proliferation was seen when MCF-Ca and LTLT cells were incubated with a series of doses of MEK1/2 inhibitor (UO126) and MAPK inhibitor (PD98059). These inhibitors had no effect on the proliferation of parental MCF-7Ca cells but caused a dose dependent inhibition of growth in LTLT cells. In addition, ER expression was restored to MCF-7Ca levels in LTLT cells treated with MEK inhibitor (PD98059) suggesting a return to hormone sensitivity.

When LTLT cells were incubated with the tyrosine kinase inhibitor, gefitinib (Iressa), there was a synergistic effect when combined with the aromatase inhibitor, anastrozole, although the cells were resistant to anastrozole alone and were only slightly inhibited by gefitinib alone. Herceptin, a clinically used antibody that blocks HER-2 caused a marked dose response inhibition of growth of LTLT cells and completely restored ER levels and sensitivity to estradiol whereas p-HER-2 and p-MAPK levels were inhibited. Herceptin had a synergistic effect when combined with letrozole and cell growth was markedly inhibited. These results suggest that sensitivity to hormones and aromatase inhibitor treatment can be restored in the tumors by blocking growth factor receptor signaling.

Combination Treatment: Letrozole and Fulvestrant-As ER appears to have an important role in the loss of sensitivity to estrogens and aromatase inhibitors, the effect of causing down-regulation of ER on MAPK activation was investigated [8]. Mice were treated with the antiestrogen fulvestrant in combination with letrozole. Although tumor growth was inhibited by fulvestrant for approximately 6 weeks and with letrozole for 17 weeks, tumor growth remained suppressed for the entire experiment of 29 weeks with the combination. The combined treatment also inhibited the expression of HER-2 and p-MAPK in the tumors.

In conclusion, our data suggests that the aromatase inhibitor letrozole was more effective than tamoxifen and fulvestrant or these agents in sequence or combination. Although letrozole was effective for significantly longer than the antiestrogens, eventually tumors began to grow despite continued letrozole treatment. The mechanism of the loss of sensitivity is associated with the activation of the MAPKinase signaling pathway. Sensitivity to estrogen and inhibition of estrogen synthesis by aromatase inhibitors can be restored in letrozole resistant cells by blocking the tyrosine kinase receptor pathway involving MAPK. This can be achieved by targeting HER-2 or by degrading the ER. Thus, it may be possible to prolong the benefits of 
aromatase inhibitor therapy and sustain well tolerated "hormone therapy" to achieve significantly longer effective treatment.

\section{References}

1. Yue W, Zhou DJ, Chen S, Brodie AMH. A new nude mouse model for postmenopausal breast cancer using MCF-7 cells transfected with the human aromatase gene. Cancer Res 1994;54:5092-5095. [PubMed: 7923123]

2. Yue W, Wang J, Savinov A, Brodie A. Effect of aromatase inhibitors on the growth of mammary tumors in a nude mouse model. Cancer Res 1995;55:3073-3077. [PubMed: 7606729]

3. Zhou D, Pompon D, Chen S. Stable expression of human aromatase complementary DNA in mammalian cells: a useful system for aromatase inhibitor screening. Cancer Res 1990;50:6949-6954. [PubMed: 2208160]

4. Lu Q, Wang J, Liu Y, Long B, Brodie A. The effects of aromatase and antiestrogens in the nude mouse model. Breast Cancer Res Treat 1998;50:63-71. [PubMed: 9802621]

5. Lu Q, Liu Y, Long BJ, Grigoryev D, Gimbel M, Brodie A. The effect of combining aromatase inhibitors with antiestrogens on tumor growth in a nude mouse model for breast cancer. Breast Cancer Res Treat 1999;57:183-192. [PubMed: 10598045]

6. Long BJ, Jelovac D, Handratta V, Thiantanawat A, MacPherson N, Ragaz J, Brodie AM. Therapeutic strategies using the aromatase inhibitor letrozole and tamoxifen in breast cancer model. J Nat Cancer Inst 2004;96(6):456-465. [PubMed: 15026471]

7. Long BJ, Jelovac D, Thiantanawat A, Brodie AM. The effect of second-line antiestrogen therapy on breast tumor growth after first-line treatment with the aromatase inhibitor letrozole: long-term studies using the intratumoral aromatase postmenopausal breast cancer model. Clin Cancer Res 2002;8:23782388. [PubMed: 12114443]

8. Jelovac D, Sabnis G, Long BJ, Goloubeva OG, Brodie AMH. Activation of MAPK in xenografts and cells during prolonged treatment with aromatase inhibitor letrozole. Cancer Res 2005;65:5380-5389. [PubMed: 15958587]

9. Pinheiro, JC.; Bates, DM. Mixed-Effects Models in S and S-Plus. Springer-Verlag; New York (NY): 2000. p. 21-45.p. 174-96.

10. Dunnett CW. A multiple comparisons procedure for comparing several treatments with control. J Am Stat Assoc 1955;50:1096-1121.

11. Scheffe H. A method of judging all contrasts in the analysis of variance. Biometrica 1959;40:87-104.

12. Baum M, Buzdar AU, Cuzick J, Forbes J, Houghton J, Klijn JGM, et al. Anastrozole alone or in combination with tamoxifen versus tamoxifen alone for adjuvant treatment of postmenopausal women with early breast cancer: first results of the ATAC randomized trial. Lancet 2002;359:21312139. [PubMed: 12090977]

13. Coombes RC, Hall E, Gibson LJ, et al. A randomized trial of exemestane after two to three years of tamoxifen therapy in postmenopausal women with primary breast cancer. N Eng J Med 2004;350:1081-1092. 

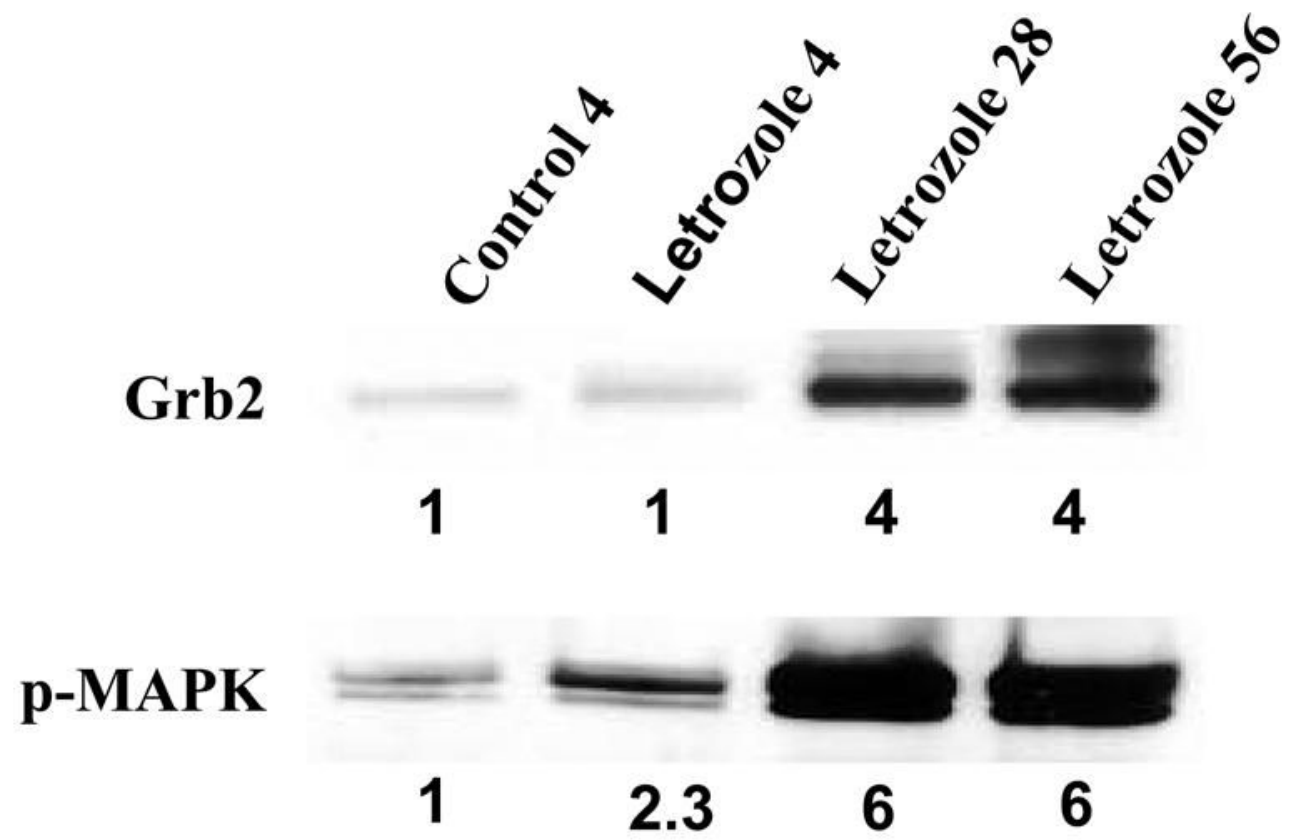

MAPK

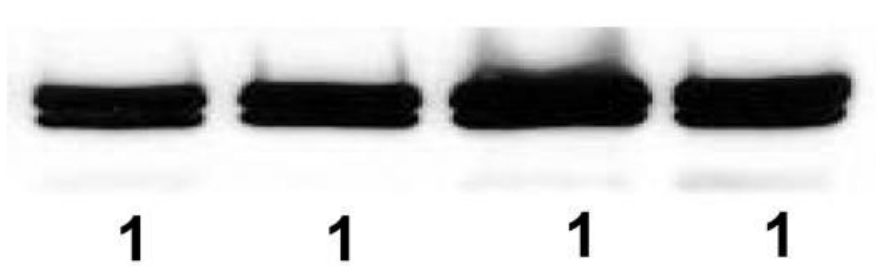

$\mathbf{p}-\mathbf{E R} \alpha$
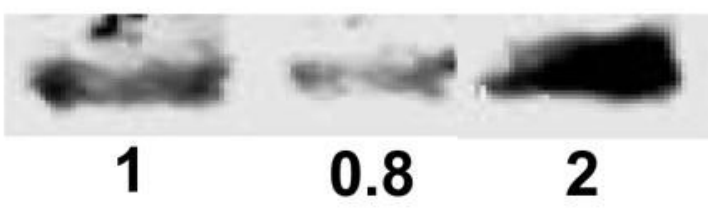

$2 \quad 2$

\section{$\beta$-actin}

Figure 1. The Effect of Letrozole Treatment on Grb2, p-MAPK, and p-ER $\alpha_{i}$ Expression in MCF-7Ca Tumor Xenografts

Tumors were collected from letrozole-treated mice at 4 weeks (when they were responding to letrozole), 28 and 56 weeks (when they were growing on letrozole), analyzed by Western immunoblotting, and were compared with tumors of vehicle-treated mice collected at week 4 (control). Tumors were homogenized in lysis buffer, and equal amounts of protein $(60 \mathrm{Ag})$ were separated on a denaturating polyacrylamide gel and transferred to a nitrocellulose membrane. After blocking nonspecific binding with 5\% nonfat milk in PBS-T, the membranes were incubated with respective primary antibodies, and specific binding was visualized by using species-specific immunoglobulin G followed by ECL detection (ECL kit) and exposure to ECL X-ray film. After exposure to X-ray film, the membranes were stripped and probed for $\beta$-actin to confirm that equal amount of proteins were loaded in each lane. Numbers below the blots represent fold change in protein expression compared with the control obtained by densitometric analysis. (From Jelovac et al., Cancer Res., 2005). 


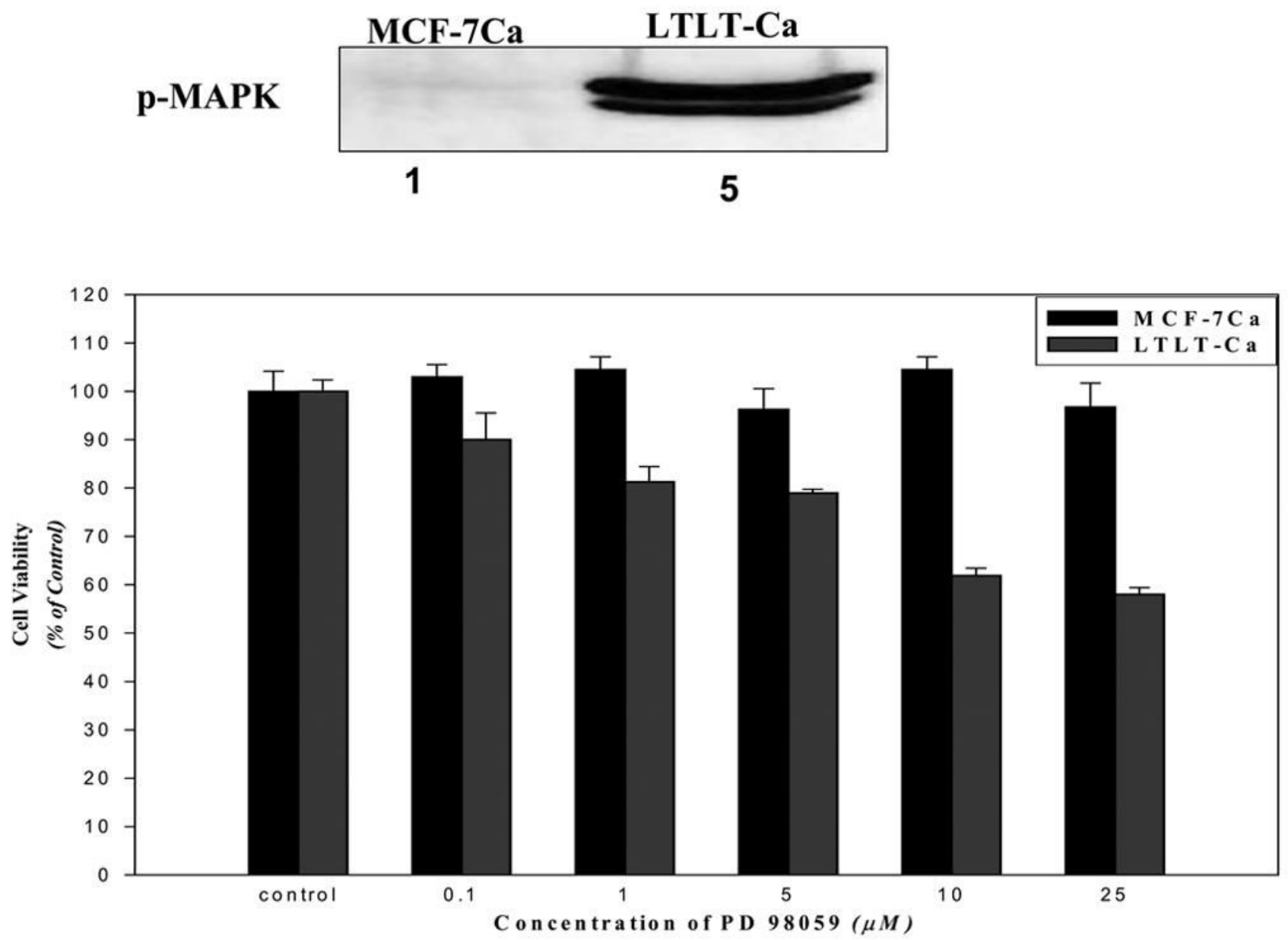

Figure 2. The Effect of MAPKinase Inhibitor, PD98059 on the Growth of MCF-7Ca and LTLTCa Cells

MCF-7Ca and LTLT-Ca cells were transferred into steroid-free medium for 3 days before plating $\left(1 \times 10^{4}\right.$ cells per well $)$ into 24 -well plates. The next day, cells were washed with DPBS and treated with steroid-free medium containing androstenedione $(25 \mathrm{nmol} / \mathrm{L})$ and the indicated concentrations of PD98059. The medium was changed every 3 days, and the cells were counted 9 days later using the MTT assay. Percentage of the PD98059 cells compared with the vehicletreated cells (control). Expression of pMAPK is shown in the insert. (From Jelovac et al., Cancer Res., 2005). 

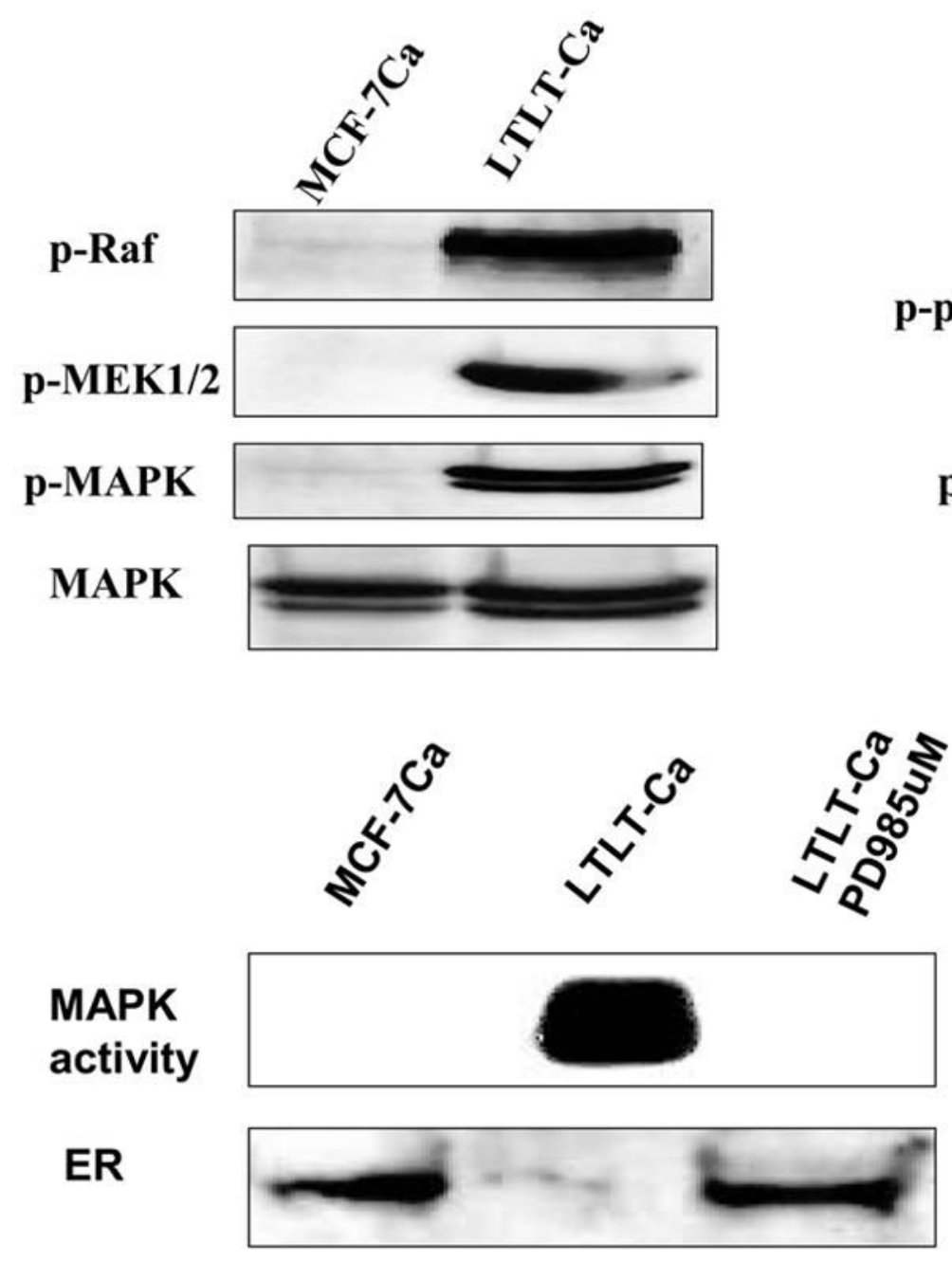

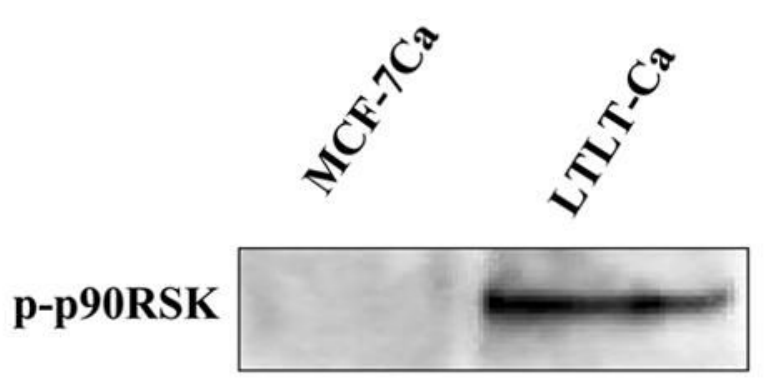

p90RSK

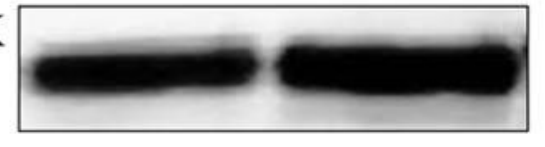

p-Elk

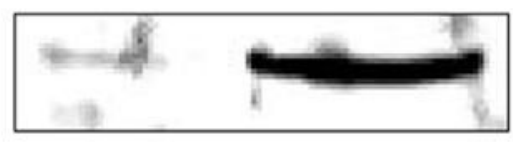

$\beta$-actin

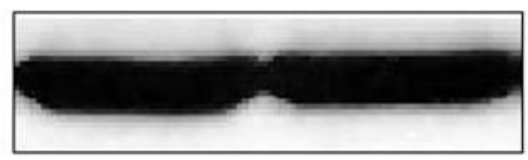

Figure 3. The Expression of Estrogen Receptor Protein and Signaling Proteins p-Raf, p-MEK1/2, p-MAPK, p-p90RSK, and p-Elk in LTLT-Ca Cells Compared with the Parental MCF-7Ca Cells Cell lysates were prepared as described in Methods. Equal amounts of protein (60 Ag) were separated on a denaturating polyacrylamide gel and transferred to a nitrocellulose membrane. After blocking nonspecific binding with 5\% nonfat milk in PBS-T, the membranes were incubated with the respective primary antibodies, and specific binding was visualized by using species-specific immunoglobulin G followed by ECL detection (ECL kit) and exposure to ECL $\mathrm{X}$-ray film. After exposure to X-ray film, the membranes were stripped and probed for h-actin to confirm that equal amount of proteins were loaded in each lane. Numbers below the blots represent fold change in protein expression compared with the control obtained by densitometric analysis. For MAPK activity, assay cell lysates were prepared as described in Methods. Proteins (200 Ag) were subjected to immunoprecipitation using specific MAPK antibody. Next day, after adding kinase reaction buffer and GST fusion protein of Elk-1 (MAPK substrate), samples were incubated at $30^{\circ} \mathrm{C}$ for 30 minutes. Samples were analyzed by Western blotting using phospho-Elk-1 antibody. (From Jelovac et al., Cancer Res., 2005). 